\title{
Leptin and Insulin Resistance in Young Adult Obese Females: Effect of Eight Weeks Resistance Training
}

\author{
Soraya Khalili ${ }^{1}$, Reza Nuri ${ }^{2}$, Mehrzad Moghadassi $^{3}$ and Mehdi Mogharnasi ${ }^{4}$ \\ ${ }^{1}$ Department of Sport Sciences, Fars sciences and Researchs center, Islamic Azad University, Shiraz, \\ Iran \\ ${ }^{2}$ Department of Sport Sciences, Kish Internation Campus, University of Tehran, Kish Island, Iran \\ ${ }^{3}$ Department of Sport Sciences, Islamic Azad University, Shiraz Branch, Shiraz, Iran \\ ${ }^{4}$ Department of Sport Sciences, University of Sistan and Balochestan, Zahedan, Iran
}

Correspondence should be addressed to: Reza Nuri; nuri_r7@yahoo.com

Received Date: 21 Augast 2013; Accepted Date: 27 January 2014; Published Date: 28 February 2014

Academic Editor: Trino J. Baptista

Copyright (C 2014 Soraya Khalili, Reza Nuri, Mehrzad Moghadassi and Mehdi Mogharnasi.

Distributed under Creative Commons CC-BY 3.0

\begin{abstract}
The aim of this study was to investigate the effect of eight weeks resistance training on leptin levels changes and insulin resistance in obese females.

Twenty inactive and obese female students (mean age $23.5 \pm 1.56$, the average height of $160.1 \pm$ 4.4 and the average BMI $31.17 \pm 1.51$ ) participated in this study.

Subjects randomly were divided into two groups: controls 10 and experimental 10. Subjects in experimental group were performed 8 weeks resistance training program (chest press, leg press, lat pull down, leg curl, bicep curl, leg extension) with $60-70$ percent of $1 \mathrm{RM}$. Data were analyzed using by analysis of covariance (ANCOVA) and independent t-test. $(\mathrm{P} \leq 0.05)$.

The results of this study showed that, there was no significance differences in leptin, insulin and glucose levels and insulin resistance between control and experimental groups $(P>0.05)$, after 8 weeks of resistance training. However, there was a significant difference between two groups $(\mathrm{P}<0.05)$ in body weight, body mass index and percent body fat. After eight weeks resistance training, body weight, BMI, WHR and percent body fat in the experimental group were decreased.
\end{abstract}

Our study showed that 8 weeks resistance training have a positive effect on reducing body weight, BMI, WHR and percent body fat but could not decrease leptin, insulin and glucose levels and insulin resistance.

Keywords: Leptin, Insulin Resistance, Resistance Training. 


\section{Introduction}

Obesity has increased in the last few decades in developed and developing countries and it has been declared a global epidemic (Sánchez-Contreras et al., 2009). More than 1.6 bilion people are overweight and 0.4 billon people suffer obesity (Janghorbani et al., 2007). It is a chronic disorder that is characterized by accumulation of fat in the body (Labib, 2003). The main function of adipose tissue is energy storage and release of free fatty acids and glycerol.

Adipose tissue plays an important role in regulating hormone and energy homeostasis (Dumortier et al., 2003). However, several mechanism have been investigated in the obesity that hormone secreted from adipose tissue such as leptin. Leptin is a protein that consists of 167 amino acids and involved in metabolic processes and represents body fat storage (Kraemer et al., 2002 and Wallace et al., 2000). Leptin is the main peptide secreted from adipose tissue that its serum concentration is an important environmental messenger in the regulation of food intake and energy expenditure (Weigle et al., 2003). The level of serum leptin correlates with the fat content of the body (Casimiro-Lopes et al., 2009, Williams et al., 2009). Significant correlation between leptin and insulin has been identified as regulators of food intake and energy balance (Nostu et al., 2007, Sharma et al., 2008). Rudzka-Kocjan et al reported that there is direct relationship between leptin and insulin resistance and fasting glucose levels, so that an increase in blood leptin concentration can lead to insulin resistance and fasting glucose increase (Rudzka-Kocjan et al., 2006). Leptin resistance can lead to insulin resistance and diabetes. This idea of leptin as an insulin- sensitizing hormone and leptin deficiency, or resistance, as a potential link between obesity and diabetes has been reviewed recently (Yildiz, 2006). The plasma leptin level has a direct relation with the fat tissue and a negative relation with the body fitness or physical activity (Courteix et al., 2007). Physiological stress resulting from physical activity is one of the potential regulators of leptin secretion from adipose tissue. Changes in the exchange of fuel, and systemic hormones concentrations and energy expenditure may be effect in plasma leptin concentrations and followed on the function of leptin (Kraemer et al., 2002).

Many researchers have been studied the effects of exercise training on leptin. Also, some authors have reported that exercise training may reduce leptin that depends on caloric intake and exercise duration but some other researchers have reported no change in leptin caused by exercise or physical activity (Gippini et al., 1999, Zafeiridis et al., 2003, Bouassida et al., 2006). A study demonstrated that leptin did not correlate with BMI in body builders and that resistance training did not influence leptin production independently of changes in body composition (Gippini et al., 1999). Kannaley et al did not observe changes in plasma leptin in diabetic subjects after acute and chronic resistance training (Kannaley et al 2001). In another study, Nindl et al measured leptin concentrations following 50 sets of resistance training. They reported no acute changes in leptin concentrations. (Nindl et al., 2002). Zafeiridis et al compared three types of resistance training protocols: (i) strength; (ii) hypertrophy; and (iii) muscular endurance, and reported significant reductions in leptin through 30 minutes of recovery. However, similar reductions were observed in nonexercising, control subjects (Zafeiridis et al., 2003).

Lambert et al demonstrated a significant increase in serum leptin after resistance training in elderly men. (Lambert et al., 2003). Fenicchia et al measured effects of resistance training ( 6 weeks, 3 sets of 8 to 12repetition) on glucose and insulin in women with type 2 diabetes. There were no significant changes in glucose or insulin levels (Fenicchia et al., 2004). Fatouros et al also, reported a decrease in plasma leptin concentration after resistance training (1year, 3 days/week, 10 exercises/three sets) in fifty inactive men (Fatouros et al., 2005). In another study, 12 obese male subjects (BMI=33.6 (1.2) kg/ m2) were investigated 
before and following 3 months of dynamic strength training. After training, total body mass, the whole-body glucose disposal rate increased by $24 \%$, and plasma levels of leptin decreased by $21 \%$ (Klimcakova et al., 2006). Lau et al were studied a short-term effects of resistance training on serum leptin levels in obese adolescents. Contrary to prior practice, the relative level of leptin was significantly reduced (Lau et al., 2010). Daryanoosh et al determined the effect of aerobic and resistance exercises (weight training) on hormonal changes in sixty non-athlete male students (Daryanoosh et al., 2010). However, leptin changes following resistance training less investigated in young adult obese females. Although, in recent years the studies have been carried out in this area, no similar findings and general conclusion has been obtained in terms of the role of resistance training on blood leptin levels in young adult obese females. Thus the aim of this study is to investigate the effect of eight weeks resistance training on plasma levels of leptin and insulin resistance in young adult obese females.

\section{Materials and Methods}

\section{Participants}

Twenty obese female students (mean age, $23.05 \pm 1.56$, BMI $31.17 \pm 1.51$ and percent fat, $27.26 \pm 1.04$ ) were selected for participation in a training program and were randomly divided into experimental group (EG: $n=10$ ) and control group (CG: $\mathrm{n}=10$ ). At baseline, all subjects were informed about the study protocol, and a written consent was provided from each participant and participate in physical activity readiness questionnaire (PAR-Q) was used. The participants were informed about the aim, advantages and possible dangers of the experimental design. This study was approved by Ethical Committee of Islamic Azad University, Fars Science and Research Center. The physical and physiological characteristics of the subjects are presented in table 1 . The entry condition to the study was the age domain between 20 and 25 years old, no history of regular exercise, lack of specific diseases and no smoking, cardiovascular disease, oral contraceptives, alcohol and menstrual disorders for at least 6 recent months. The amount of calories the subjects using records to three-day food questionnaires and was implemented at the beginning, middle and end of the training program. Both groups were advised to observe their usual diet during the period of study.

\section{Anthropometric Measurements}

The weight and height of the participants were measured with thin clothes and without shoes by using the standard hospital scales. Body Mass index (BMI) was calculated using the formula body weight $/$ height $^{2}$ in terms of $\mathrm{kg} / \mathrm{m}^{2}$ (Eizadi et al., 2011). Abdominal circumference and hip circumference were measured. Also, Waist to hip circumference ratio (WHR) was assessed through dividing the abdominal circumference by hip circumference. The Subcutaneous fats of participants were measured by using the caliper and technique of pinch in the triceps, the pelvis and hip (three sites method). Measurement was taken from the right side of body for three times within 20 seconds between each period for to recovery and was placed in the Jackson Pollock formula to calculate percent body fat. To omit individual error, all the measurements were done by one staff.

\section{Resistance Training Program}

The participants were completed an eight week program of resistance training on three days of the week. Each session lasted about one hour, which included a 10minute warm-up, 40 minutes of weight training and a 10-minute cool-down. The program followed a training design with the $60-70 \%$ of their 1-repetition maximum (1RM) with 3 sets of 8 to 10 repetitions for all exercises. Muscle strength was measured with the 1-RM testing method. Strength was recorded as the maximal weight lifted in one full range of motion, and the 1-RM was determined after either four or five trials. Strength testing on the following six exercise stations: chest press, leg press, Lat pull down, leg curl, bicep curl, leg extension that include upper and lower body limbs (Saremi A and Parastesh M., 
2011). To observe the principle of overload, again a repetition maximum was measured at weeks 4 and 8. Principle of overload was designed to after 4 weeks of training. 1RM for each individual test was performed at each station and the weights were adjusted based on it. The control group was asked does not doing any exercise or weight loss program during the 8 weeks. It should be noted that during the three-day food questionnaire survey of the early was implemented in middle and end of the training. The control group had no exercise training during eight weeks.

\section{Biochemical Measurements}

After 12 to $14 \mathrm{~h}$ of fasting, $10 \mathrm{ml}$ of venous blood was collected from each participant while they were seated and resting and were in the follicular phase of the menstrual cycle (9-3 days after menstruation). The plasma was separated by a $3000 \mathrm{rpm}$ centrifuge and was stored at a fridge with the temperature of $-80^{\circ} \mathrm{C}$ until the day of the experiment. At endpoint, after 48 hours of the last training session (to remove the effect of training), blood samples were taken from both groups. Fasting plasma leptin was calculated using the ELISA method, competitive sandwich type (kit of Diagnostics Biochem Canada Inc, with sensitivity of $0.5 \mathrm{ng} \mathrm{mL}$ ). The levels of insulin were calculated using the ELISA method, competitive sandwich type (kit of Monobind Inc Company, America; sensitivity of $0.5 \mu \mathrm{UI} / \mathrm{ml}$ ). Glucose levels were assessed using the photometric method. Insulin resistance index (Homeostasis Model Assessment Insulin Resistance-HOMA-IR) was calculated based on the multiplication of the fasting blood insulin concentration $(\mathrm{mu} / \mathrm{ml})$ by the fasting glucose concentration $(\mathrm{mg} / \mathrm{dL})$ divided by the constant of 405 (Matthews et al 1985).

\section{Statistical Analysis}

Results were expressed as the mean \pm SD and distributions of all variables were assessed for normality. First, Kolmogorov-
Smirnov test was used for normality of the data distribution. Paired t-test was used to compute mean ( \pm SD) changes in the variables in control and training group pre and after the intervention. A two-way ANCOVA was used to determine if significant $(\mathrm{P}<0.05)$ differences existed among the groups for leptin, insulin, glucose and insulin resistance variables between before and after exercise training. Post-test was the dependent variable, group was the fixed factor and pre-test was the covariate. The relationships between changes variables between groups and over time were determined using Pearson's correlation test .Significant level was set as $\mathrm{p} \leq 0.05$. All data were analyzed using by the SPSS software (Version 18).

\section{Result}

The results of this study showed that 8 weeks of resistance training significantly decrease BMI $(31.32 \mathrm{~kg} / \mathrm{m} 2$ versus 29.73 $\left.\mathrm{kg} / \mathrm{m}^{2}, \mathrm{P}=0.0001\right)$, Weight Body $(80.5 \mathrm{~kg}$ versus $76.25 \mathrm{~kg}, \mathrm{P}=0.0001)$, WHR $(0.93$ versus0.89, $\mathrm{P}=0.0001)$ and body fat percent (27.48 versus 24.85, $\mathrm{P}=0.0001$ ) in $\mathrm{EG}$. Whereas in the CG there was significant increase in BMI, Weight Body, WHR and body fat percent. Statistically significant differences were not seen in leptin $(p=0.939)$, Insulin $(p=0.336)$, Glucose $(p=0.264)$ and Insulin resistance $(P=0.306)$ between CG and EG. (Table1). Pearson's correlation demonstrated a positive relationship between plasma leptin levels with insulin resistance $(\mathrm{r}=0.08, \mathrm{P}=0.8)$ and body fat percentage $(\mathrm{r}=0.23, \mathrm{P}=0.5)$ but did not achieve statistical significance.

All data were not significant for normality check. Data of carbohydrate, fat and protein consumption and calorie intake of subjects during eight weeks are given in Table 2. Result showed that the subjects maintained their dietary habits throughout the study. 
Table1. Anthropometric and Metabolic Characteristics of Participants in Experimental and Control Group in Pre and Post Test

\begin{tabular}{|c|c|c|c|c|c|}
\hline & \multicolumn{2}{|c|}{ Experimental group } & \multicolumn{2}{|c|}{ Control group } & \multirow[b]{2}{*}{$P$ - value } \\
\hline & Pre test & post test & Pre test & ost test & \\
\hline Age (years) & $23.8 \pm 1.61$ & & & $23.1 \pm 1.52$ & \\
\hline Height $(\mathrm{cm})$ & $160.3 \pm 4.83$ & & & $159.9 \pm 3.97$ & \\
\hline Weight(kg) & $80.5 \pm 4.87$ & $76.25 \pm 5.14^{*}$ & $79.35 \pm 4.91$ & $82.35 \pm 4.37$ & 0.0001 \\
\hline $\mathrm{BMI}\left(\mathrm{kg} / \mathrm{m}^{2}\right)$ & $31.32 \pm 1.39$ & $29.73 \pm 1.42^{*}$ & $31.02 \pm 1.63$ & $32.05 \pm 1.58$ & 0.0001 \\
\hline WHR & $0.93 \pm 0.04$ & $0.89 \pm 0.05 *$ & $0.92 \pm 0.03$ & $0.96 \pm 0.02$ & 0.0001 \\
\hline Fat $(\%)$ & $27.48 \pm 1.03$ & $24.85 \pm 1.53^{*}$ & $27.05 \pm 1.06$ & $28.59 \pm 1.02$ & 0.0001 \\
\hline Leptin(mg/l) & $56.46 \pm 29.01$ & $41.25 \pm 28.01$ & $52.92 \pm 24.37$ & $54.02 \pm 15.37$ & 0.939 \\
\hline Insulin $(\mu \mathrm{u} / \mathrm{ml})$ & $14.76 \pm 7.18$ & \begin{tabular}{|l|}
$13.59 \pm 7.33$ \\
\end{tabular} & $14.57 \pm 7.28$ & $16.23 \pm 4.4$ & 0.336 \\
\hline $\begin{array}{l}\text { Insulin } \\
\text { resistance }\end{array}$ & $3.19 \pm 1.66$ & $2.78 \pm 1.65$ & $3.12 \pm 1.96$ & $3.45 \pm 1.29$ & 0.306 \\
\hline Glucose(mg/dl) & $85.7 \pm 1.034$ & $80.3 \pm 12.1$ & $83.3 \pm 11.4$ & $86.3 \pm 17.45$ & 0.264 \\
\hline
\end{tabular}

Data are as means \pm SE. $\quad *:(\mathrm{p}<0 / 05)$

Table 2. The Composition of the Subjects' Diets (Carbohydrate, Fat and Protein) and Calorie Intake during 8 Weeks (Mean \pm SD)

\begin{tabular}{|l|l|l|}
\hline & Control group & Experimental group \\
\hline Carbohydrate $(\mathrm{g})$ & $467.5 \pm 45.7$ & $499.4 \pm 47.8$ \\
\hline Fat $(\mathrm{g})$ & $77.9 \pm 15.7$ & $79.9 \pm 12.1$ \\
\hline Protein (g) & $112.5 \pm 9.7$ & $114.2 \pm 9.4$ \\
\hline Energy intake (kcal) & $3121.1 \pm 29.7$ & $3173.5 \pm 22.1$ \\
\hline
\end{tabular}

Data are the mean \pm SE of carbohydrate, fat and protein consumption and calorie intake of subjects in each group. Result showed that the subjects maintained their dietary habits throughout the study.

\section{Discussion}

The aim of the present study was to investigate the effects of resistance training on leptin and insulin resistance. The results of this study demonstrated that eight weeks resistance training reduce body weight, BMI, WHR but it can't change leptin, insulin and glucose levels and insulin resistance, significantly. Because of small sample size, we had type II error. In accordance with our results, Gippini et al, (1999), Zafeiridis et al (2003), Fenicchia et al (2004), Bouassida et al (2006), Lau et al (2010) did not observe significant changes in serum leptin and insulin levels following resistance training. Our findings aren't in agreement with findings of Fatouros et al (2005) and Daryanoosh et al (2010). Fatouros et al (2005) demonstrated that plasma leptin concentration decrease after resistance training and they observed a significant relationship between decreased leptin and reduced insulin. Probably, this inconsistence is realated to duration of exercise program (1-year) and decreased insulin. Insulin may regulate and control the involved leptin. Also, in study of Daryanoosh et al (2010) after 8-weeks (Balk field test), leptin and insulin levels decreased, significantly. They showed that there was a positive significant relationship between decreased leptin and insulin levels. Kraemer et al believed that differences in the populations investigated (men and women, trained and untrained), exercise training protocols used (intensity, volume, type and duration), and participants conditions and energy balance might be contributing to these conflicting results on the relationship between leptin concentrations and exercise training (Kraemer et al 2002).

In recent years, several studies evaluated the effects of strength training on leptin levels in several populations and presented conflicting data regarding the behavior of this variable (Mota, 2007). Since in this study body mass index and percent body 
fat significantly were decreased, we expected to reduce significantly plasma leptin levels. According to studies, the reduction of leptin has been attributed to alteration in energy balance, improvements in insulin sensitivity, alteration in lipid metabolism and lipid concentration. Exercise training and physical activity can reduce fat mass, play a critical role in energy expenditure and affect hormonal concentrations and metabolites. Also, exercise training could modify the leptin response. It seems that this response is related to several factors, such as intensity and the duration of the exercise training, nutritional status of the participants, circadian rhythm of leptin, the time of blood sampling and caloric imbalance affected by the exercise (Bouassida, 2006). Another proposed mechanism is related to energy expenditure during exercise training. Several studies suggest that generally short-term exercises $\quad<60$ minutes) or physical activity that generated energy expenditure lower than 800 Kcals do not modify the concentrations of leptin (Kraemer et al., 2002). Decreased leptin concentration after a long-term exercise $(\geq$ $60 \mathrm{~min}$ ) has been attributed to diurnal reduction in circulating leptin and hormonal changes induced by exercise (Bouassida 2006). Another reason for the lack of significant reduction in leptin may be related to the increased amount of cortisol during the training program. Some researchers have reported that it may increase the amount of cortisol simultaneously with the effects of exercise on leptin (Zafeiridis et al., 2003). However, due to limited resources, the amount of these hormones was not measured in the subjects in this study. In this study, 8 weeks of resistance training had no significant effect on insulin levels and insulin resistance. Possible mechanisms is decreased insulin, increased glucose transport protein (GLUT4), reduced secretion and increased free fatty acids, increased delivery of glucose to the muscles and changes in muscle tended to increase glucose availability (Irwin et al., 2005).

This study had such limitation such as small sample size, no follow-up study and no measurements of daily physical activity in two groups during eight weeks.

The high-intensity resistance exercise training increases glucose uptake and glycogen is depleted. In this study, another reason to expect a reduction in fat mass is related to insulin that the rate of $6.9 \%$ decrease. It seems that fat mass is associated with insulin levels. So, reduced fat mass, also vary insulin levels. Perhaps, lack of significant amounts of leptin is related to the nutrition status and its type. High intensity and long term exercise probably by increasing the muscle mass, skeletal muscle glucose transport protein by increasing or decreasing the synthesis of fatty acids, insulin sensitivity and glucose uptake by skeletal muscle activity increases. One reason may be lack of meaningful resistance exercise on insulin levels and insulin resistance in the present study is exercise intensity and duration.

\section{Conclusions}

The present study demonstrated that 8 weeks resistance training have a positive effect on reducing body weight, BMI, WHR and percent body fat but could not decrease leptin, insulin and glucose levels and insulin resistance. Based on these results, it could be suggested that further research is recommended to examine the effects of resistance training on leptin and insulin resistance with control of factors such as lifestyle or daily energy expenditure, diet or without it, cortisol and hormonal concentrations.

\section{Acknowledgements}

The authors would like to thank the University of Darab Students for their commitment in this study.

\section{References}

Bouassida, A., Zalleg, D., Bouassida, S., et al. (2006). "Leptin, its Implication in Physical Exercise and Training: A Short Review," Journal of Sports Science and Medicine, 5 172-181.

Casimiro-Lopes, G., Oliveira-Junior, A. V., Portella, E. S., Lisboa, P. C., Donangelo, C. M., 
De Moura, E. G. \& Koury, J. C. (2009). "Plasma Leptin, Plasma Zinc, and Plasma Copper are Associated in Elite Female and Male Judo Athletes," Biological Trace Element Research, 127(2) 109-115.

Courteix, D., Rieth, N., Thomas, T., Van Praagh, E., Benhamou, C. L. \& Collomp, K. (2007). "Preserved Bone Health in Adolescent Elite Rhythmic Gymnasts despite Hypoleptinemia," Hormone Research. 68(1) 20-7.

Daryanoosh, F., Mehrabani, G. \& Shikhani, H. (2010). "The Effect of Aerobic and Resistance Exercises on Hor-monal Changes in Non-Athlete Students at Shiraz University, Southern Iran," Iranian Red Crescent Medical Journal 12(2) 127-132.

Dumortier, M., Brandou, F., Perez-Martin, A., et al. (2003). "Low Intensity Endurance Exercise Targeted for Lipid Oxidation Improves Body Composition and Insulin Sensitivity in Patients with the Metabolic Syndrome," Diabetes \& Metabolism, 29 509-18.

Eizadi, M., Shafiei, M., Sedaghati, S. \& Jenabi, A. (2011). "Serum Leptin and Nsulin Resistance are Not Affected by an Acute Cycling Exercise in Diabetic Patients," Indian Journal of Fundamental and Applied Life Sciences, 1 (4) 364-370.

Fatouros, I. G., Tournis, S., Leontsini, D., Jamurtos, A. Z., et al. (2005). "Leptin and Adiponectin Responses in Overweight Inactive Elderly Following Resistance Training and Detraining are Intensity Related," The Journal of Clinical Endocrinology and Metabolism, 90 59705977.

Fenicchia, L. M., Kanaley, J. A., Azevedo, J. L., Miller, C. S., Weinstock, R. S., Carhart, R. L. \& Ploutz-Snyder, L. L. (2004). "Influence of Resistance Exercise Training on Glucose Control in Women with Type 2 Diabetes," Metabolism, 53(3) 284-289.

Gippini, A., Mato, A., Peino, R., et al. (1999). "Effect of Resistance Exercise (Body Building) Training on Serum Leptin Levels in Young Men Implications for Relationship between Body Mass Index and Serum Leptin," Journal of Endocrinological Investigation, 22 824-28.

Irwin, M. L., Mc Tiernan, A., Bernstein, L., et al. (2005). "Relationship of Obesity and Physical Activity with C-Peptide, Leptin, and Insulin-Like Growth Factors in Breast Cancer Survivors," Cancer Epidemiology, Biomarkers \& Prevention, 14 2881-2888.

Janghorbani, M., Amini, A., Willett, W. C., Gouya, M. M., Delavari, A., Alikhani, S. \& Mahdavi, A. (2007). "First Nation Wide Survey of Prevalence of Overweight, Underweight, and Abdominal Obesity in Iranian Adults," Obesity, 15 2797-2808.

Kanaley, J. A., Fenicchia, L. M., Miller, C. S., et al. (2001). "Resting Leptin Responses to Acute and Chronic Resistance Training in Type 2 Diabetic Males and Females," International Journal of Obesity \& Related Metabolic Disorders, 25, 1474-1480.

Klimcakova, E., Polak, J., Moro, C., et al. (2006). "Dynamic Strength Training Improves Insulin Sensitivity without Altering Plasma Levels and Gene Expression of Adipokines in Subcutaneous Adipose Tissue in Obese Men," The Journal of Clinical Endocrinology \& Metabolism, 91 5107-12.

Kraemer, K. K., Chu, H. \& Castracane, V. D. (2002). "Leptin and Exercise," Experimental Biology and Medicine (Maywood), 227 (9) 701-708.

Labib, M. (2003). "The Investigation and Management of Obesity," Journal of Cinical Pathology, 56 17-25.

Lambert, C. P., Sullivan, D. H. \& Evans, W. J. (2003). "Effects of Testosterone Replacement and/or Resistance Training on Interleukin-6, Tumor Necrosis Factor A, and Leptin in Elderly Men Ingesting Megestrol Acetate: A Randomized Controlled Trial," The Journals of Gerontology. Series A, Biological Sciences and Medical Sciences, 58A 165-170.

Lau, P. W. C., Kong, Z., Choi, C.- R., et al. (2010). "Effects of Short-Term Resistance 
Training on Serum Leptin Levels in Obese Adolescents," Journal of Exercise Science \& Fitness, 8(1) 54-60.

Matthews, D. R., Hosker, J. P., Rudenski, A. S., Naylor, B. A., Treacher, D. F. \& Turner, R. C. (1985). "Homeostasis Model Assessment: Insulin Resistance and $\beta$-cell Function from Fasting Plasma Glucose and Insulin Concentrations in Man," Diabetologia, 28, 412-9.

Mota, G. R. \& Zanesco, A. (2007). "Leptina, Ghrelina E Exercício Físico," Arquivos Brasileiros de Endocrinologia \& Metabologia, 51(1) 25-33.

Nindl, B. C., Kraemer, W. J. \& Arciero, P. J. (2002). "Leptin Concentrations Experience a Delayed Reduction after Resistance Exercise in Men," Medicine and Science in Sports and Exercise, 34 608-13.

Nostu, Y., Nabika, T., Shibata, H. \& Nagai, A. (2007). "HOMA-IR and Related Clinical Parameters," The Japanese Journal of Clinical Pathology, 55(8) 737-742.

Rudzka-Kocjan, A., Szarras-Czapnik, M. \& Ginalska-Malinowska, M. (2006). "Estimation of the Correlation of Insulin Resistance and Selected Adipocytokines in Children with Simple Obesity--Preliminary Study," Endokrynologia, Diabetologia $i$ Choroby Przemiany Materii Wieku Rozwojowego, 12(3) 211-15.

Sánchez-Contreras, M., Moreno-Gómez, G. A., Marín-Grisales, M. E. \& Factores De Riesgo (2009). "Cardiovascular en Poblaciones Jóvenes," Revista de Salud Pública, 11(1) 110-122.
Saremi, A. \& Parastesh, M. (2011). "TwelveWeek Resistance Training Decreases Myostatin Level and Improves Insulin Sensitivity in Overweight-Obese Women," International Journal of Diabetes and Metabolism, 19 63-68.

Sharma, M. D., Garber, A. J. \& Farmer, J. A. (2008). "Role of Insulin Signaling in Maintaining Energy Homeostasis," Endocrine Practice, 14(3) 373-380.

Wallace, A. M. (2000). "Measurment of Leptin Binding in the Human Circulation," Annals of Clinical Biochemistry, 37 244-252.

Weigle, D. S., Cummings, D. E., Newby, P. D., Breen, P. A., et al. (2003). "Roles of Leptin and Ghrelin in the Loss of Body Weight Caused by a Low Fat, High Carbohydrate Diet," The Journal of Clinical Endocrinology \& Metabolism, 88 1577-1586.

Williams, K. W., Scott, M. M. \& Elmquist, J. K. (2009). "From Observation to Experimentation: Leptin Action in the Mediobasal Hypothalamus," The American Journal of Clinical Nutrition, 89(3) 985-990.

Yildiz, B. O. \& Haznedaroglu, I. C. (2006). "Rethinking Leptin and Insulin Action: Therapeutic Opportunities for Diabetes," The International Journal of Biochemistry \& Cell Biology, 38. 820 -30.

Zafeiridis, A., Smilios, I. \& Considine, R. V. (2003). "Serum Leptin Responses after Acute Resistance Exercise Protocols," Journal of Applied Physiology, 94 591-97. 